Physica B 165\&166 (1990) 1219-1220

North-Holland

\title{
NERNST EFFECT IN A HIGH TEMPERATURE SUPERCONDUCTOR
}

\author{
Hans LENGFELLNER, Andreas SCHNELLBÖGL, Josef BETZ, Wilhelm PRETTL and Karl Friedrich RENK \\ Institut für Angewandte Physik, Universität Regensburg, 8400 Regensburg, W.Germany
}

\begin{abstract}
A Nernst effect has been observed in a Tl-Ba-Ca-Cu-O superconducting thin film. The film was irradiated with laser pulses inducing a temperature gradient perpendicular to the film surface. A Nernst voltage was obtained perpendicular to both the temperature gradient and a magnetic field applied parallel to the film surface. We attribute the Nernst effect to flux depinning and flux transport driven by the laser induced thermal gradient. From an analysis of the dependence of the Nernst voltage on film temperature we find a distribution of pinning energies ranging from $100 \mathrm{~K}$ to $4000 \mathrm{~K}$.
\end{abstract}

Flux motion and flux pinning in high temperature superconductors currently is a main issue of many investigations (1-5). We report here on a new method for studying flux dynamics. The method is based on the Nernst effect, which is observed for the first time in a high temperature superconductor.

The experiments are performed with polycrystalline superconducting $\mathrm{Tl}-\mathrm{Ba}-\mathrm{Ca}-\mathrm{Cu}-\mathrm{O}$ films (6) with a thickness of about $1 \mu \mathrm{m}$ and with $\mathrm{T}_{c}$ values near $100 \mathrm{~K}$. A bridge-shaped film (Fig. 1, inset) is irradiated with light pulses (100 ns duration, wavelength $10 \mu \mathrm{m}$ ) of a TEA$\mathrm{CO}_{2}$ laser. Radiation is absorbed in a thin $(\approx 0.1 \mu \mathrm{m})$ surface layer of the film which gives rise to a temperature gradient perpendicular to the film surface. Perpendicular to both the temperature gradient and a magnetic field applied parallel to the film surface, a time dependent Nernst voltage is observed (Fig. 1). The temperature gradient decays in a time of several $\mu$ s by thermal diffusion. The Nernst voltage is reversed in polarity if the magnetic field, obtained from small solid state magnets (field strength $\sim 1 \mathrm{kG}$ ), is reversed.

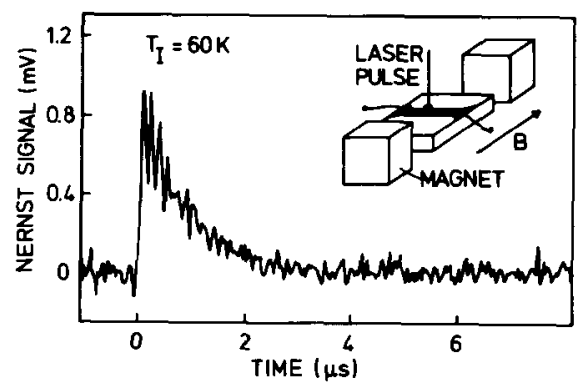

FIG. 1 - Experimental arrangement and time resolved Nernst signal, at initial film temperature $T_{I}=60 \mathrm{~K}$, and laser pulse energy density $E_{P}=6.5 \mathrm{~mJ} / \mathrm{cm}^{2}$.

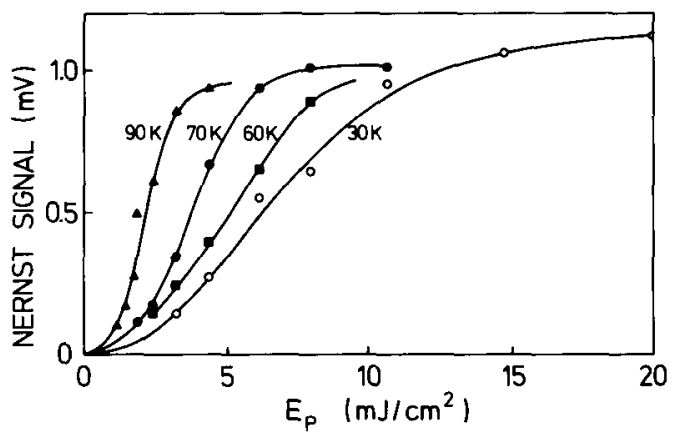

FIG. 2 - Signal height vs irradiation energy density $E_{P}$, for various initial film temperatures $T_{I}$.

In Fig. 2 the height of the Nernst signals, depending on laser energy, is shown for several initial film temperatures $T_{I}\left(\mathrm{~T}_{I}\right.$ is the film temperature before laser irradiation). With increasing $T_{I}$ the curves show a stronger increase. For high laser energies, $E_{P}$, the signals saturate because the film becomes normal conducting.

The Nernst voltage is generated by flux motion between two contact points (7). The voltage is proportional to the rate at which flux crosses a curve joining the contacts. The rate depends on the fluxon velocity which is proportional to the thermal force $f_{t h}=-S \nabla T$ (8) where $S$ is the fluxon transport entropy and $\nabla T$ is the temperature gradient, and on the depinning probability, i.e. the thermal activated hopping rate $\nu=$ $\nu_{o} \exp \left(-T_{P} / T_{F}\right)$ of fluxons. In this expression $\nu_{o}$ is the attempt frequency, $T_{P}$ the pinning energy and $T_{F}$ the film temperature.

Because the fluxons move from the hot film surface to the colder film backside and because this motion depends upon flux depinning in this colder region of the film, we chose for $T_{F}$ a film temperature near the interface between film and substrate. For small laser 


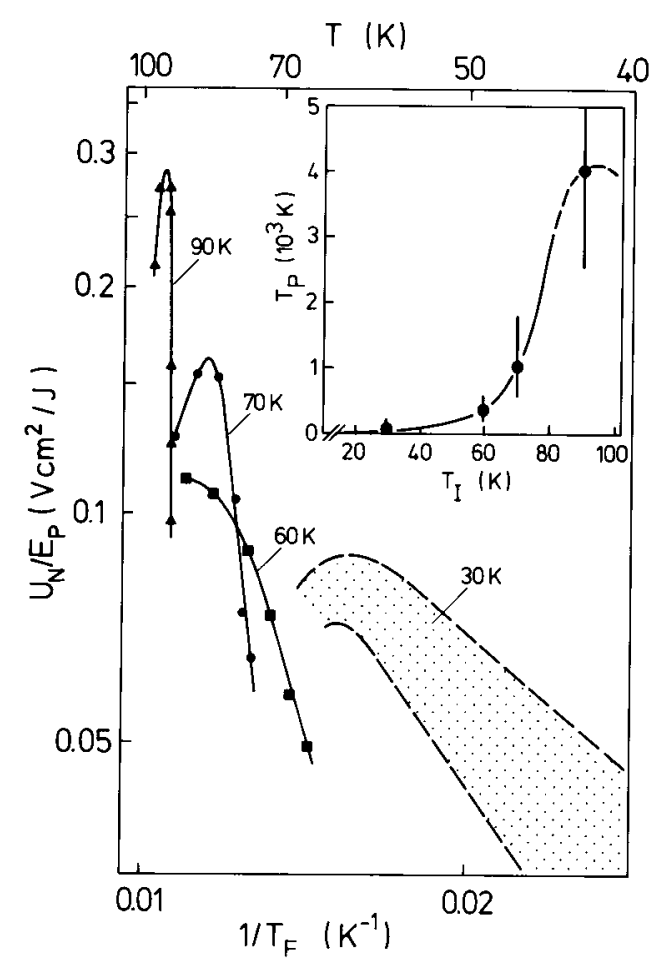

FIG. 3 - Dependence of the normalized Nernst signal $U_{N} / E_{P}$ on film temperature $T_{F}$, for various initial film temperatures $T_{I}$. The inset shows the obtained dependence of pinning energy $T_{P}$ on $T_{I}$.

pulse energies, as long as specific heat and diffusivity are constant, the film temperature rises proportional to the laser energy. From a heat transfer model (9), a measurement of the diffusivity of our film (10) and diffusivity values of $\mathrm{Y}-\mathrm{Ba}-\mathrm{Cu}-\mathrm{O}$ films (11), we obtain for our film $\Delta T=\nabla T \cdot d=A\left(T_{I}\right) \cdot E_{P}$ and $T_{F}=B\left(T_{I}\right) \cdot E_{P}+T_{I}$ where $\Delta T$ is the temperature difference between film surface and film backside, $d$ the film thickness, $E_{P}$ the energy density of the laser pulse, $T_{I}$ the initial film temperature, and $A=1.9 \times 10^{3} \mathrm{Kcm}^{2} / \mathrm{J}$, $B=3.5 \times 10^{3} \mathrm{Kcm}^{2} / \mathrm{J}$ at $T_{I}=60 \mathrm{~K}$, for example. These values change not much for higher $T_{I}$ values; for low temperatures it should be taken acount of the strongly changing specific heat. We will describe our results using an expression

$$
\begin{aligned}
U_{N} & =C \cdot \nabla T \cdot S \cdot \nu_{o} \exp \left[-T_{P} / T_{F}\right] \\
& =C^{\prime} \cdot E_{P} \cdot \exp \left[-T_{P} \cdot\left(B \cdot E_{P}+T_{I}\right)^{-1}\right]
\end{aligned}
$$

for the Nernst signal voltage $U_{N}$ with proportionality constants $C, C^{\prime}$, and with the relations given above for $\nabla T$ and $T_{F}$ for small $E_{P} . S$ is assumed to be constant.

A logarithmic plot of $U_{N} / E_{P}$ against $1 / T_{F}$ for various values of $T_{I}$ is shown in Fig. 3. At small laser energies the curves show constant slopes from which we deduce values for $T_{P}$. With increasing laser energy, $T_{F}$ increases and the curves bend due to saturation of the signal. Due to uncertain $T_{F}$ for the $T_{I}=30 \mathrm{~K}$ measurement, we give an estimated region for the slope of this curve (Fig. 3). The inset shows values of $T_{P}$ for the different $T_{I}$. Our results indicate a distribution of pinning centers with different pinning energies ranging from low values $(\$ 100 \mathrm{~K}$ ) up to values of about $4000 \mathrm{~K}$. At low temperatures only loosely bound fluxons are depinned and contribute to the signal. At higher temperatures also strongly bound flux is thermally activated leading to a strong increase of the Nernst signal.

In conclusion, we have observed, for the first time, a Nernst effect in a high temperature superconductor. An analysis of the Nernst signals based on flux motion driven by thermal forces and thermally activated hopping delivers values of the pinning energy $T_{P}$. We find, for a Tl-Ba-Ca-Cu-O thin film, a distribution of pinning energies ranging from $\lesssim 100 \mathrm{~K}$ to several thousand $\mathrm{K}$.

\section{ACKNOWLEGEMENTS}

The work was supported by the EC and by the Bundesministerium für Forschung und Technologie.

( 1) T.T.M. Palstra, B. Batlogg, R.B. van Dover, L.F. Schneemeyer, and J.V. Waszczak, Appl. Phys. Lett. 54 (1989) 763.

(2) P. Fischer, H.W. Neumüller, B. Roas, H.F. Braun, and G. Saemann-Ischenko, Solid State Comm. 72 (1989) 871.

(3) A. Gupta, P. Esquinazi, H.F. Braun, and H.W. Neumüller, Phys. Rev. Lett. 63 (1989) 1869.

(4) K.C. Woo, K.E. Gray, R.T. Kampwirth, J.H. Kang, S.J. Stein, R. East, and D.M. McKay, Phys. Rev. Lett. 63 (1989) 1877.

(5) L.J. Swartzendruber, A. Roitburd, D.L. Kaiser, F.W. Gayle, and L.H. Bennett, Phys. Rev. Lett. 64 (1990) 483.

(6) H. Lengfellner, J. Betz, and K.F. Renk, Appl.Phys. A48 (1989 501 .

(7) B.D. Josephson, Physics Letters 16 (1965) 242.

(8) R.P. Huebener, Magnetic Flux Structures in Superconductors, Springer Series in Solid-State Sciences 6, eds. M. Cardona, P. Fulde, and H.-J. Queisser, Berlin (1979).

(9) H.S. Carlslaw and J.C. Jaeger, Conduction of Heat in Solids, Clarendon Press, Oxford (1959).

(10) A. Schnellbögl, H. Lengfellner, and W. Prettl, to be published.

(11) L. Gomes, M.M.F. Vieira, S.L. Baldochi, N.B. Lima, M.A. Novak, N.D. Vieira Jr., and S.P. Morato, J. Appl. Phys. 63 (1988) 5044. 